\title{
Dictynna
}

Dictynna

Revue de poétique latine

$6 \mid 2009$

Varia

\section{Der Held und die Versuchung des weiblichen Anblicks}

Andrea Harbach

\section{OpenEdition}

1 Journals

Édition électronique

URL : http://journals.openedition.org/dictynna/279

DOI : 10.4000/dictynna.279

ISSN : 1765-3142

Référence électronique

Andrea Harbach, «Der Held und die Versuchung des weiblichen Anblicks », Dictynna [En ligne], 6 | 2009, mis en ligne le 26 novembre 2010, consulté le 10 septembre 2020. URL : http:// journals.openedition.org/dictynna/279; DOI : https://doi.org/10.4000/dictynna.279

Ce document a été généré automatiquement le 10 septembre 2020.

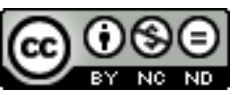

Les contenus des la revue Dictynna sont mis à disposition selon les termes de la Licence Creative Commons Attribution - Pas d'Utilisation Commerciale - Pas de Modification 4.0 International. 


\title{
Der Held und die Versuchung des weiblichen Anblicks
}

\author{
Andrea Harbach
}

1 Am Ende von Dion von Prusas erster Rede über das Königtum wird erzählt, wie sich Herakles zwischen Basileia und Tyrannis habe entscheiden müssen. ${ }^{1} \mathrm{Zu}$ Grunde liegt offensichtlich die Geschichte, die in der Antike Prodikos zugeschrieben wird, ${ }^{2}$ und zufolge der Herakles, gerade erwachsen geworden, zwischen Tüchtigkeit (Arete) und Schlechtigkeit (Kakia) wählen muss. Doch während in den bekanntesten Versionen dieser Erzählung die Frauengestalten im Redewettstreit Herakles für sich zu gewinnen suchen, kommen in Dions Adaption weder Basileia noch Tyrannis zu Wort. Bei ihm muss der junge Held seine Entscheidung vielmehr aufgrund der äußeren Erscheinung beider Frauen treffen. Dies ist eine überraschende Neuerung: Die Übertragung des Gleichnisses auf eine Wahl zwischen Formen der Herrschaft ändert an sich nichts am rationalen Anspruch der Entscheidung. Man könnte also meinen, dass Herakles auch bei dieser Wahl durchdenken muss, welche Vorzüge und Nachteile die Optionen haben, dass er die Frage nacheinander unter persönlichen, moralischen und politischen Gesichtspunkten betrachtet, um sich schließlich $\mathrm{zu}$ einer differenzierten Stellungnahme durchzuringen. Von all dem ist in Dions Text nichts zu lesen. Dort gründet Herakles seine Entscheidung einzig und allein auf die ausgiebige Betrachtung des Erscheinungsbildes der beiden Damen.

2 Dions Text ist nicht die einzige Erzählung einer Wahl, in der das Aussehen der beiden Optionen, personifiziert als Frauen, alleiniges oder zumindest hauptsächliches Kriterium für die Entscheidung wird. Ziel meines Artikels ist es, diese Konzentration auf das Visuelle in Versionen der Geschichte von Herakles' Wahl zu erklären, indem ich den Mythos vom Parisurteil heranziehe. Meine Absicht ist es, wechselseitige Einflüsse zwischen beiden Mythen aufzuzeigen, insofern sie als „Lebenswahl“-Geschichten gestaltet sind.

3 Den Inhalt der Geschichte von Herakles' Wahl, wie sie uns bei Xenophon in maßgeblicher Form überliefert ist, ${ }^{3}$ werde ich im Wesentlichen als bekannt voraussetzen. Ich werde zunächst auf das Verhältnis der visuellen Schilderung beider 
Frauen und ihrer Redebeiträge, wie es sich in Xenophons Fassung findet, eingehen. Davon ausgehend, werde ich die Tendenzen einiger Versionen aufzeigen, das Visuelle übermäßig stark in den Vordergrund zu rücken (I). Dabei werde ich die Funktion des Mythos von Herakles als „Wahl“ in Frage stellen und ihn stattdessen als Schilderung einer Versuchungssituation deuten (II). In beiderlei Hinsicht werde ich zeigen, dass im Mythos vom Parisurteil eine Wahlsituation ähnlich konzipiert wird. Den Eindruck, dass beide Geschichten nicht nur einander ähneln, sondern sich tatsächlich gegenseitig geprägt haben, möchte ich bestätigen, indem ich darüber hinaus weitere Einflüsse dieses Mythos auf Dions Version von Herakles' Wahl aufzeige (III).

In Xenophons Wiedergabe von Prodikos' Gleichnis sind Arete und Kakia durch chiastische Verteilung ihrer Qualitäten und Schwächen voneinander unterschieden : Die eine ist gut, aber weniger schön ; die andere ist schön, aber nicht gut. Der Vorzug in moralischer Hinsicht geht mit einem Mangel an Attraktivität einher, und umgekehrt. So ergibt sich ein maximaler Kontrast zwischen den beiden Optionen. Kakias erotische Anziehungskraft und Aretes Sprödigkeit werden dem Leser dadurch vermittelt, dass Aussehen und Auftreten beider Frauen beschrieben werden. Die Gestaltung ihres Erscheinungsbildes ist offensichtlich anhand der Stereotype von Matrone und Hetäre entworfen $; 4$ darüber hinausgehend, lassen sich ihre Schilderungen jedoch in mehreren Hinsichten symbolisch verstehen : Zum einen sind in ihrer Haltung, Gestik und Mimik Charaktereigenschaften kodiert ( eine derartige Auffassung, dass im Auftreten einer Person sich ihr Wesen manifestiere und es nur entschlüsselt werden müsse, war unter sophistischen Rednern sehr verbreitet. ${ }^{5}$ Als zweites spielt die Dichotomie von Schein und Sein eine wichtige Rolle bei der Kontrastierung der Frauengestalten. Die von Kakia verwendete Schminke

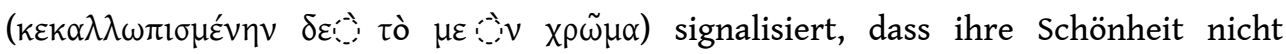

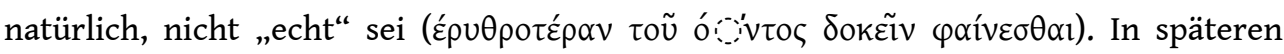
Versionen heißt es bisweilen, dass sie keine „wahre“ Schönheit besitze. ${ }^{6}$

5 Doch nicht alle Details der Beschreibung lassen sich mit diesen beiden Ansätzen erklären. Kakias transparentes Kleid $(\varepsilon \dot{\varepsilon} \sigma \tilde{\eta} \tau \alpha \delta \varepsilon$

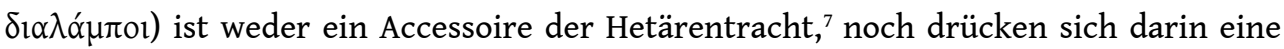
bestimmte Charaktereigenschaft oder gar unnatürlicher Schein aus. Vielmehr zielt dieses Element ihrer Aufmachung darauf ab, ihre Erotik als offensiv zu charakterisieren ; Kakia stellt eine Versuchung dar.

Der deskriptive Abschnitt der Geschichte in Xenophons Wiedergabe ist entscheidend, wenn es darum geht, das Dilemma zu eröffnen. Doch die anschließenden Reden rücken das zurecht; entscheidend ist, wie sich Kakia und Arete rhetorisch bewähren. Dem Visuellen kommt in dieser Geschichte nur eine eingeschränkte Rolle : Betrachtet man, wie viel Raum ihrer äußerlichen Beschreibung (\$ 22) und wie viel ihren Reden ( $\S \S$ 23-33) zugestanden wird, ergibt sich ein Verhältnis von 1 :11.

7 Die Geschichte hat einen enormen Erfolg erlebt und ein reiches Nachleben begründet. ${ }^{8}$ Mal wird Prodikos zitiert und der Inhalt seiner Geschichte referiert ; 9 oder das Gleichnis wird auf einen anderen Zusammenhang übertragen, wobei Kakia und Arete durch andere Personifikationen ersetzt und Herakles durch einen anderen Protagonisten abgelöst werden können. ${ }^{10}$ Auch an Parodien mangelt es nicht; bald 
schon gibt es Schilderungen, in denen ein Sprecher zweier Frauen ansichtig wird, die eine so züchtig wie die andere anziehend, und sich erwartungsvoll für die Attraktivere entscheidet. ${ }^{11}$ Zentral ist immer die Synkrisis zweier weiblicher Figuren; bereits die Agone zwischen zwei als Frauen gestalteten Personifikationen in Aristophanes' Wolken und in Kratinos' Pytine könnten erste Reaktionen auf dieses Element darstellen, das man wohl auch für Prodikos' Darbietung als gesichert annehmen darf. ${ }^{12}$ Doch während dort wie in Xenophons Fassung die wesentliche Betätigung der Frauengestalten in einem Redewettstreit besteht, fallen innerhalb der Tradition einige Adaptionen der Geschichte auf, die auf verbale Beteiligung der Personifikationen verzichten. Dions eingangs erwähnte Version ist nicht der einzige Fall. Dasselbe Merkmal liegt in den Fassungen von Maximos von Tyros, Philostrat, zwei Rhetoren des vierten Jahrhunderts und in der des Themistios vor. Auch in die Tabula Cebetis ist die Geschichte von Herakles' Wahl eingegangen und hat dort in der Konzeption von Pseudopaideia und wahrer Paideia Spuren hinterlassen. Der Fall dieser Schrift ist besonders interessant. Sie gibt sich als Deutung eines Bildes aus, das in einem Kronos-Tempel hänge und in symbolischer Form das menschliche Leben abbilde. Durch die Gestaltung als Ekphrasis ist die Wirkweise der Personifikationen von vorne herein auf die visuelle Rezeption eingeschränkt.

\section{II}

8 Doch auch wenn sich weitere Beispiele dafür finden lassen, dass die Synkrisis allein visuell vollzogen wird, sticht Dions Version unter ihnen in besonderer Weise heraus. Die Geschichte ist als eine Art platonischer Mythos an den Schluss der Rede gesetzt, um die im Vorangegangenen entfalteten politischen Thesen zu illustrieren und so noch einmal zu bekräftigen. Dion, als Sprecher der Rede, verbürgt sich selbst für den Wahrheitsgehalt des Mythos: Auf einer seiner Wanderungen sei er vom Weg abgekommen und an einem alten Heraklesheiligtum auf eine alte Frau getroffen, die ihn ihm erzählt habe. ${ }^{13}$

Die Wahl läuft in seiner Fassung so ab, dass Hermes zu Herakles gesandt wird, als Zeus erkennt, dass das politische Interesse seines Sohnes erwacht. Der Götterbote führt den jungen Helden zu zwei Gipfeln, auf dem zwei Frauen sitzen, nämlich Basileia und Tyrannis. Ausführlich begutachten sie die Wege, die zu den Gipfeln heraufführen, den Lebensraum der beiden Frauen, sowie diese selbst und ihre Begleiterinnen. Als Hermes Herakles im Anschluss auffordert, sich für eine von ihnen zu entscheiden, fällt dieser seine Entscheidung sehr dezidiert zu Gunsten von Basileia (§ 83).

10 Die ausführliche Beschreibung aller Details der Frauengestalten und ihrer Gipfel nimmt nicht wie bei Xenophon einen, sondern dreizehn Paragraphen ein; sie erstreckt sich über §§ 66-72 und 77-82. Was zwischen den beiden Abschnitten liegt, gibt sich zwar als Gespräch zwischen Herakles und seinem Führer aus, und ist in der Tat dialogisch gestaltet. Doch inhaltlich geht es auch in diesen Paragraphen wieder um den Anblick, der sich ihnen bietet. Hermes zeigt, benennt und erklärt, was sie sehen. Die wörtliche Rede dient allein dazu, in Form von Hermes' Erläuterungen Aspekte sichtbar werden zu lassen, die Herakles sonst nicht hätte sehen können : So identifiziert er die Begleiter und Begleiterinnen von Basileia als Personifikationen ihr verwandter Werte (§ 74-75). Er erklärt, welche Art von Menschen zu Tyrannis' Gipfel strebe (§ 76). Seine Rede dient 


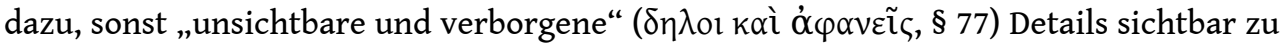
machen.

11 In Dions Fassung wird die Beschränkung der Personifikationen auf ihre visuelle Rolle nicht nur praktiziert, sondern auch explizit thematisiert. Auffällig ist die Häufung von Verben, die visuelle Wahrnehmung beschreiben :

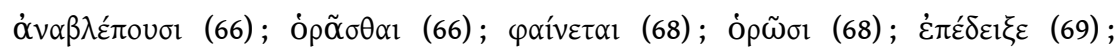

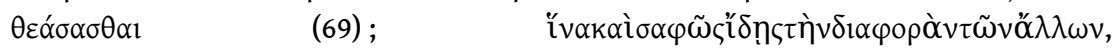

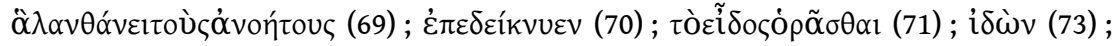

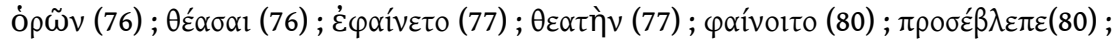

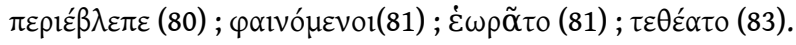

12 Als sich Herakles am Ende der Geschichte entscheiden muss, wird seine Wahl in direkte Beziehung zu dem Anblick gestellt: Er äußert seinen Entschluss, „als er dies alles

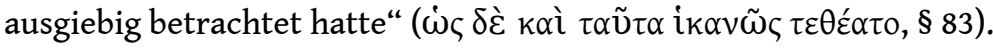

Am deutlichsten wird die Eingrenzung von Herakles' Rolle auf die eines Betrachters, als es um des Weg geht, der zu Tyrannis' Gipfel hinaufführe. Diesen habe er natürlich nicht betreten, heißt es von dem jungen Herrscher, der sich nichts hat zu Schulden kommen lassen. Doch es ist nicht allein dieser Nebensinn intendiert, den wir auch heute verstehen, wenn davon die Rede ist, dass man nie einen schlechten Weg eingeschlagen

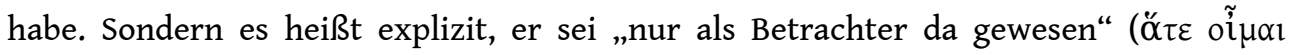

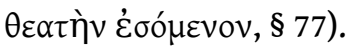

Tatsächlich tritt Herakles nicht nur insofern als Betrachter auf, dass er, wie beschrieben, alles genau inspiziert. Sondern die ganze Geschichte erscheint wie die Beschreibung eines Bildes; der Blick des Lesers wird gelenkt durch den Blick des Betrachters Herakles. Dreimal wechselt die Perspektive auf den Anblick, und zwar wird dies dadurch erreicht, dass Herakles in der Geschichte $\mathrm{zu}$ einem anderen Beobachtungsposten geht. Am Anfang führt Hermes ihn auf einen erhabenen Ausblick

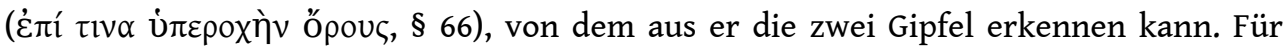
Betrachter hingegen, die von unten in die Höhe blicken, scheint es sich um die Spitze

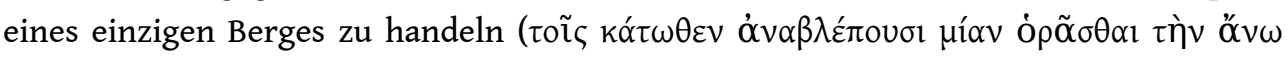

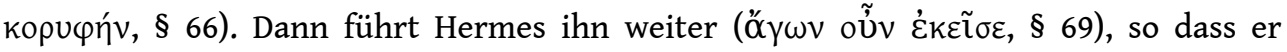
Basileia im Detail betrachten kann, und anschließend zu einem dritten Punkt, von wo aus er dasselbe mit Tyrannis tun kann. Die Geschichte von Herakles' Wahl ist in dieser Fassung auf komplexeste Weise visualisiert : Die Geschichte besteht hauptsächlich aus Beschreibungen der Personifikationen; die rein visuelle Entscheidungsfindung wird mehrfach thematisiert ; und der geschilderte Anblick bietet sich wie ein Kunstwerk da, das sich aus verschiedenen Blickwinkeln betrachten lässt.

Welche Absicht könnte mit dieser Visualisierung verfolgt sein ? Wenn man bedenkt, um was für eine Art von Entscheidung es sich hier handelt - nämlich um eine fundamentale politische Orientierung -, mutet es doch erstaunlich an, dass die Wahl allein anhand visuellen Inputs getroffen wird. Aber handelt es sich wirklich um eine Wahl?

Diese Frage kann man bereits an die prodikeische Urfassung stellen : Dort lässt sich beobachten, dass die Alternativen eigentlich alles andere als gleichwertig sind. Betrachtet man Aussehen und Wesen zugleich, besitzen zwar beide Frauen einen Vorzug und eine nachteilige Eigenschaft. Doch bei der Wahl, die Herakles zu treffen hat, geht es allein um eine moralische Entscheidung, nicht um eine ästhetische. Unter diesem Gesichtspunkt jedoch ist Arete ihrer Gegnerinbei weitem überlegen; deren 
Schönheit stellt lediglich ein Moment der Ablenkung da in der ansonsten a priori eindeutig definierten Gewichtung. Bereits die Namen „Tüchtigkeit“ und "Schlechtigkeit" zeigen, dass an ihrer Bewertung kein Zweifel gelassen wird. Herakles' Wahl erzielt also keineswegs einen Erkenntnisgewinn; denn seine Entscheidung wirft kein neues Licht auf die Optionen. Vielmehr steht er selbst im Fokus der Bewertung: Lässt er sich von Kakias Schönheit trügen oder bleibt er standhaft und hält an seinem Wissen um den moralischen Wert beider Frauen fest ? Seine Wahl sagt in erster Linie etwas über ihn selbst aus. Er entscheidet sich für die Tüchtigkeit, denn er ist ein tüchtiger Held, so suggeriert die Geschichte. Bei der Begegnung mit Kakia und Arete handelt es sich also um eine Probe, bei der der Held seine moralische Standfestigkeit unter Beweis stellen muss. Tatsächlich wurde beobachtet, dass die Sprache der Verführung eine große Rolle spielt in der Tradition von Herakles' Wahl. ${ }^{14}$ Die „Wahl“ ist eine Prüfung, der der Held unterzogen wird, eine Versuchung.

Deutlich zu beobachten ist dies an einer Parodie von Herakles' Wahl, die eben diesen Effekt nutzt. In einem Fragment des Komikers Alexis fordert Linos, der mythische Erzieher des Herakles, seinen Schüler auf, sich ein Buch auszuwählen. ${ }^{15}$ Er bietet ihm Werke verschiedenster Gattungen und Autoren an: Orpheus, Hesiod, Tragödien, Choirilos, Homer, Epicharm und allerlei andere Schriften. Dann fügt er seiner Aufforderung zur Wahl hinzu : Denn damit wirst du dein wahres Ich zeigen, wonach dir am

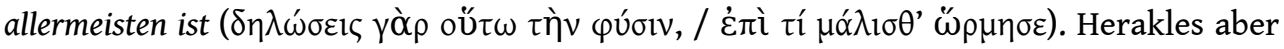
fühlt sich zu keiner der intellektuell anspruchsvollen Lektüren hingezogen, sondern entscheidet sich für ein - Kochbuch. Der sonst so standhafte Held wird in der parodistischen Verzerrung zum Opfer der (in diesem Falle : kulinarischen) Versuchung. Was Linos mit seiner Warnung an Herakles zum Ausdruck bringt, ist genau die Tatsache, dass bei der Wahl letztendlich nicht die Beurteilung der Optionen im Vordergrund steht, sondern die Entscheidung auf denjenigen zurückfällt, der sie trifft, und mehr über ihn selbst aussagt als über das Objekt seiner Wahl.

Aus der 1. Person geschildert, kann das Modell von Herakles' Wahl deshalb genutzt werden, um sich selbst darzustellen. Ovid lässt am Anfang des dritten Buches der Amores seinen poeta zwischen zwei personifizierten Gattungen wählen; indem er Tragoedia (noch) zurückweist und sich für Elegia entscheidet, signalisiert er, Elegiker zu sein. Ähnlich schildert Lukian einen Traum, den er in seiner Jugend gehabt habe. Darin seien ihm Hermoglyphike, die Steinmetzkunst, und Paideia, die Bildung, erschienen, die ihn beide für sich hätten gewinnen wollen. Wie es der Rolle des Sprechers entspricht, der die Geschichte seinem Publikum vorträgt, hat er sich am Ende des Traumes für Paideia entschieden. ${ }^{16}$ Die Handlung klingt zwar in der Zusammenfassung eindeutig, wird aber in der ausführlichen Gestaltung in mehrerlei Hinsicht unterlaufen. Literarische Anspielungen auf berühmte trügerische Träume deuten an, wie sehr sich seine Wahl tatsächlich bewährt habe $;{ }^{17}$ das Ausmaß der glorreichen Zukunft, die er sich weiter erträumt, lassen weitere Zweifel aufkommen. ${ }^{18}$ Vor allem aber spielt diese Wahl mit widersprüchlichen Entsprechungen zwischen den beiden Personifikationen und ihren prodikeischen Vorbildern. Paideia, die Siegerin, tritt wie Arete an zweiter Stelle im Agon an. Doch äußerlich ähnelt sie eher Kakia; denn sie ist bei weitem

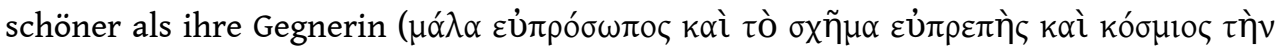
$\dot{\alpha} v \alpha \beta 0 \lambda \eta ́ v, \S 6)$. In der Tat wird ihr Aussehen keinen unwesentlichen Einfluss haben auf die Entscheidungsfindung. Zwar bleibt es nicht allein bei der Beschreibung der beiden Frauengestalten, wie dies bei Dion der Fall war; sondern die beiden halten durchaus 
Reden, mit denen sie den jungen Mann von sich zu überzeugen suchen. Aber er hört gar nicht bis zum Ende hin, sondern fällt seine Entscheidung, bevor die Reden beendet

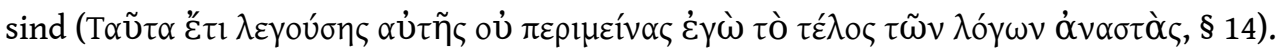
So ist es dann auch die „Hässliche, Männliche“, die er zurücklässt (

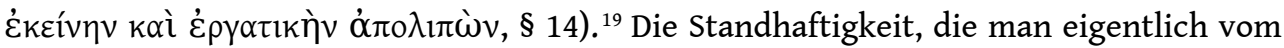
jungen Helden angesichts seiner Entscheidung erwartet, wird hier parodiert. Der Antiheld verfällt hoffnungslos dem Schönen und widersteht keine Sekunde lang der Versuchung. Zugrunde liegt aber dasselbe Schema: Die Wahl befördert nicht neue Erkenntnis über die Bewertung der Berufsbilder Bildhauer und Redner zutage ; sondern es handelt sich um einer Versuchungssituation, der sich der Held ausgesetzt sieht.

III bestehen gilt, muss sich auch bei Dion der junge Held erst bewähren, bevor es ihm gestattet ist, sein politisches Interesse in die Tat umzusetzen. Herakles steht abgesehen von parodistischen Umsetzungen - exemplarisch für den Helden, der sich in einer solchen Situation als standhaft erweist. Gegenteilig verhält es sich mit einem anderen mythischen Helden, der ebenfalls einer Wahlsituation ausgesetzt war :20 Als Paris beim Wettstreit der drei Göttinnen Aphrodite zur Schönsten kürte, löste dieses Urteil nicht nur den Trojanischen Krieg aus. ${ }^{21}$ In ihm manifestierte sich auch Paris' Charakter, der in der Tradition immer als unkriegerisch und der Liebe zugeneigt geschildert wird. ${ }^{22}$ Besonders explizit wird der Zusammenhang zwischen dem Schiedsrichter des Schönheitswettbewerbs und seiner Entscheidung bei Apuleius formuliert. In seinen Metamorphosen wohnt Lucilius einer Aufführung des Parisurteils als Mimus bei. ${ }^{23}$ Zunächst noch begeistert vom lasziven Auftritt der Darstellerin der Venus, macht er plötzlich seiner Verärgerung über „Richter wie Paris“ Luft. ${ }^{24}$ Die Korruption heutiger Richter sei kein Wunder, wenn man bedenke, dass doch schon beim Inbegriff eines Urteilsspruchs Paris, „dieser Bauer und Hirte, vom Ratschluss des großen Jupiter zum Richter ernannt (iudex electus), zum Profit seiner Libido verschachert" habe. ${ }^{25}$

Der iudex wird in dieser Tirade zum Beurteilten. Dies ist eine naheliegende Deutung des Parisurteils; doch sie ist nicht notwendigerweise Bestandteil jeder Darstellung dieses Mythos. Er auch ohne weitere Deutung als Schönheitswettbewerb repräsentiert werden ; gerade in bildlichen Darstellungen scheint dies oft der Fall gewesen zu sein, da dort die Göttinnen zunächst überhaupt nicht differenziert wurden und somit keinen Anhaltspunkt für eine Unterscheidung in verschiedene „Optionen“ einer Wahl boten. ${ }^{26}$ Nur in einigen der uns überlieferten Versionen scheint eine moralisierende Lesart als Wahl zwischen drei Lebensweisen wirklich angebracht zu sein. ${ }^{27}$

Immer jedoch gilt Paris' Entscheidung für Venus als Beispiel einer schlechten Wahl. Nie wird in Betracht gezogen, Paris für seine Entscheidung für die Liebe Anerkennung zu zollen. Wie anders die Lehre ausfallen kann, die aus demselben Stoff gezogen wird, zeigt eine indische Adaption des Parisurteils. Im Padmavati Natak sieht sich der König Indranil wie Paris ebenfalls drei schönen Göttinnen gegenüber; er trifft genau wie sein okzidentaler Vorfahre seine Entscheidung zugunsten von Rati, einer Art ,Aphrodite', und erhält dafür die schönste Frau : Jedoch endet an dieser Stelle die indische Version der Geschichte mit Heirat und Happy End..$^{28}$ 
In der griechisch-römischen Tradition stellt die Geschichte vom Parisurteil jedoch, wie gesagt, eine Versuchungssituation dar, die sich komplementär zu der des Herakles verhält. Während letzterer die Probe besteht und insofern eine, gute` Wahl trifft, weiß Paris der Versuchung nicht zu widerstehen und trifft also eine ,schlechte Wahl. Die strukturelle Ähnlichkeit beider Mythen wurde bereits von Athenaios konstatiert : „Ich aber sage, dass auch das Urteil des Paris früher schon zu einer Wahl der Lust auf Kosten der Tugend gemacht wurde. Dadurch dass freilich Aphrodite der Vorzug gegeben wird - und sie verkörpert eben die Lust -, gerät alles aus dem Gleichgewicht. Und mir scheint, dass unser guter Xenophon die Geschichte von Herakles und Arete genau danach gemacht hat. “29

Dem Mythos vom Parisurteil ist in besonderer Weise eine visuelle Dimension zu eigen. ${ }^{30}$ Unabhängig von seiner Deutung als Entscheidung zwischen drei Gaben, die die Göttinnen Paris anbieten und die ihm eine unterschiedliche Zukunft eröffnen, besteht die Handlung in erster Linie in einem Schönheitswettbewerb; die Göttinnen präsentieren ihr Aussehen dem Schiedsrichter. Das Parisurteil war eines der beliebtesten Motive auf Vasenmalereien in der Antike. ${ }^{31}$ In der Literatur animierte der Auftritt der Göttinnen immer wieder $\mathrm{zu}$ detaillierten pornographischen Beschreibungen. ${ }^{32}$ Das erotische Potenzial des Mythos darf als sehr groß angesehen werden; tatsächlich war er sehr beliebt in erotischen Epigrammen. Die Adaptionen spitzen den pornographischen Charakter des Stoffes häufig noch zu, beispielsweise wenn die drei Göttinnen durch drei (detailliert beschriebene) weiblichen Gesäßen oder drei (noch detaillierter beschriebene) Vulven ersetzt werden, die es zu vergleichen gilt. ${ }^{33}$ Was bei Herakles' Wahl durch Kakias Stilisierung zu einer verführerischen Frau erreicht wird, ist beim Parisurteil von vornherein vorhanden : Sexuelle Versuchung ist ein unabdingbarer Bestandteil dieses Mythos.

Die Tatsache aber, dass das Parisurteil sich so offensichtlich zu visueller Repräsentation anbietet, legt nahe, dass sich gerade auch visuell gestaltete Versionen von Herakles' Wahl besonders durch den anderen Mythos haben inspirieren lassen. Der Verzicht auf wörtliche Reden der Frauengestalten und die komplexe Gestaltung ihrer Betrachtung durch Herakles erinnern an Paris' Wahl. Die Entscheidung, die Herakles zu treffen hat, wird dadurch noch weiter als Versuchungssituation zugespitzt; selbst unter dem rein visuellen Eindruck muss bei Herakles die Ratio die Oberhand behalten.

In der Tat lassen sich in Dions Version von Herakles' Wahl noch andere Elemente entdecken, die traditionell zum Parisurteil gehören. Zum einen ist die Handlung an einen anderen Ort verlegt, als man es aus früheren Versionen findet. Gewöhnlich assoziiert man Herakles' Wahl mit einer Weggabelung, an der ihm die zwei Frauen begegnet seien. Bei Dions hingegen sind die Personifikationen auf zwei Berge versetzt. Ein Gebirge als Umfeld kennt man aber gerade vom Parisurteil, dessen Vollstreckung gewöhnlich am Ida situiert wird. ${ }^{34}$ Des weiteren ist für den Vollzug der Wahl eine zusätzliche Figur eingeführt, nämlich Hermes. In früheren Versionen von Herakles' Wahl existiert er nicht; dort ist Herakles allein mit den beiden Frauengestalten. Bei Dion ist ihm der Götterbote an die Seite gestellt, der von Zeus gesandt wurde und die Aufgabe hat, die Wahl zu moderieren (§ 65-66) : Er führt Herakles zu verschiedenen Aussichtspunkten, erklärt den Anblick, benennt die Frauen, fordert ihn schließlich zur Wahl auf und referiert Herakles' Entscheidung an Zeus (§ 83). Im Parisurteil ist Hermes immer zugegen; wie gerade für Dions Version beschrieben, ist er dort der Tradition zufolge der Moderator des Wettstreits. Von Zeus beauftragt, geleitet er die Göttinnen 
$\mathrm{zu}$ ihrem Richter und sorgt dort für die Ausführung der Wahl. Seine Präsenz in literarischen Darstellungen wie auf Abbildungen ist notorisch, selbst in Fällen, wo sie dramatisch nicht erforderlich ist. Fast nie wird seine Gestalt in Repräsentationen des Mythos weggelassen. ${ }^{35}$ Des weiteren findet sich ein wichtiger Anhaltspunkt in der Beschreibung von Basileia, bei der nämlich auf ihre Ähnlichkeit mit Hera verwiesen

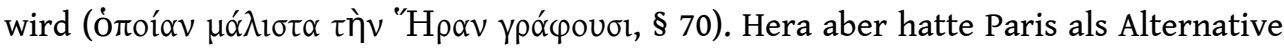
zu Aphrodites Angebot der schönsten Frau vielen Darstellungen zufolge Macht als Herrscher angeboten. ${ }^{36}$

Bezeichnenderweise begegnen auch in den anderen Versionen von Herakles' Wahl, in denen wie in Dions auf Reden der Frauen verzichtet wird, diese Elemente, die an Repräsentationen des Parisurteils erinnern. In der Tabula Cebetis, bei Themistios und zwei Rhetoren sitzen die Frauengestalten ebenfalls auf Bergen. Bei den zwei Rhetoren wird die Wahl noch dazu von Hermes geleitet; bei Themistios wird Herakles von Phronesis geführt, einer allegorischen Personifikation, die aber offensichtlicht die gleiche Funktion erfüllt : den Anblick zu deuten und den 'Richter' zur Entscheidung zu führen. In den Versionen der Geschichte hingegen, in denen der Redeagon zwischen den beiden Kontrahentinnen beibehalten wird, ist weder von Bergen, noch von Hermes die Rede. Tatsächlich scheint Herakles' Wahl in der Antike kein beliebter Bildstoff gewesen zu sein. ${ }^{37}$

Dions Gestaltung von Herakles' Wahl greift offensichtlich eine bestehende Verwandtschaft mit dem Parisurteil auf und betont die Ähnlichkeit noch stärker. Darüber dürfen natürlich nicht die Elemente, die in Dions Version eindeutig der Tradition von Herakles' Wahl entstammen, aus dem Blick geraten : Abgesehen von der Figur des Herakles an sich und der Zweizahl der Optionen, die sich ihm bieten, deuten das noch jugendliche Alter des Helden (\$ 66) darauf hin, sowie der große Raum, den die Wegmetaphorik einnimmt ( $\$ 67$ und 77). Die Anklänge an das Parisurteil ersetzen nicht die eine Wahl durch die andere, sondern bewirken eine bestimmte Ausrichtung in der Gestaltung des Mythos, nämlich eine Zuspitzung der Versuchung.

\section{BIBLIOGRAPHIE}

J. Alpers (1912), Hercules in bivio, Göttingen.

F. Buffière (1973), Les mythes d'Homère dans la pensée grecque, Paris.

R. Cribiore (2007), „Lucian, Libanius, and the short road to rhetoric“, GRBSt 47, S. 71-86.

M. Davies (1981), „The judgement of Paris and Iliad Book XXIV“, JHS 101, S. 56-62.

M. Davies (2003), „The judgement of Paris and Solomon“, CQ ns.53, S. 32-43.

M. Davies (2004), „The temptress throughout the ages : further versions of Heracles at the crossroads“, CQ ns. 54, S. 606-610.

J. Elsner (2007), Roman eyes. Visuality and subjectivity in art and text, Princeton/Oxford. 
E. Finkerpearl (1991), „The judgement of Lucius : Apuleius, Metamorphoses 10.29-34“, CA 10, S. 221-236.

J.T. Fitzgerald und L.M. White (1983), The tabula of Cebes, Chico.

O. Gigon (1956), Kommentar zum zweiten Buch von Xenophons Memorabilien, Basel.

M. Gleason (1995), Making men. Sophists and self-presentation in ancient Rome, Princeton.

H. Gomperz (1912), Sophistik und Rhetorik. Das Bildungsideal des eu legein in seinem Verhältnis zur Philosophie des fünften Jahrhunderts, Leipzig.

G.B. Kerferd (1954-1955), „The relativism of Prodicus“, BRL 37, S. 249-256.

A. Kossatz-Deissmann (1994), „Paridis Iudicium“, in LIMC Bd. 7, Zürich, S. 176-188.

M. Kuntz (1993-1994) : „The Prodicean Choice of Herakles. A reshaping of myth“, CJ 89, S. 163-181.

J. Mesk (1934), „Dion und Themistios“, Philologische Wochenschrift 54, S. 556-558.

J. Moles (1990), „The kingship orations of Dio Chrysostomos“, in : F. Cairns und M. Heath (Hrsgg.), Papers on the Leeds International Latin Seminar Bd. 6, Leeds, S. 297-375.

D. Page (1978), The epigrams of Rufinus. Edited with an introduction and commentary, Cambridge.

E. Panofsky (1930), Hercules am Scheidewege und andere antike Bildstoffe in der neueren Kunst. Leipzig/ Berlin.

E. Stafford (2005) : „Vice or virtue? Heracles and the art of allegory“, in Bowden/Rawlings (Hrsgg.) : Herakles and Hercules. Exploring a Graeco-Roman divinity, Llandysul, S. 71-96.

T.C.W. Stinton (1965), Euripides and the judgement of Paris, London.

T. Whitmarsh (2001), Greek literature and the Roman empire. The politics of imitations. Oxford.

M. Zimmerman-de Graaf (1993) : „Narrative judgement and reader response in Apuleius' Metamorphoses 10.29-34 : the pantomime of the judgement of Paris“, Groningen colloquia on the novel 5, S. 143-161.

\section{NOTES}

1. Dio Chrys. Or. 1.59-83.

2. Zitiert wird Prodikos als Autor der Geschichte bei Xen. Mem. 2.1.21, Schol. ad. Arist. Nub. 361, Maxim. Tyr. 14.1, Philostr. VA 6.10, Athen. 510C, Themist. Or. 22 280a-282c. Identifiziert mit Xenophon nennt ihn auch Cic. Off. 1.118.

3. Xen. Mem. 2.1.21-40. Auf die Frage, inwiefern es sich dabei tatsächlich um eine getreue Wiedergabe von Prodikos ' Version handelt, möchte ich an dieser Stelle nicht weiter eingehen, da sie für die hier betrachtete Frage nicht relevant ist

4. Gigon (1956) 64-65.

5. Gleason (1995) 55-81.

6. Max. Tyr. 14.1, negativ formuliert über ihre Gegnerin bei Philo De sacr. 21.

7. Gigon (1956) 64-65.

8. Siehe Alpers (1912), Panofsky (1930).

9. Cic. Off. 1.118, Max. Tyr. 14.1, Them. 280a, Rhet. Troil. VI p. 52 Walz, Rhet. ignot. herausg. bei Rabe (1909).

10. Tab. Ceb. 24-25, Ov. Am. 3.1, Philo De sacr. 20-45, Sil. Pun. 15.18-128, Dio Chrys. Or. 1.59-83, Lukian. 32.6-11, Philostr. VA 6.10-11, Themist. 280b-282c. 
11. Philod. Ep. 38 Siders, Ov. Am. 3.1, Lukian. 35.

12. Gomperz (1912) 91. Siehe auch Kerferd (1954-55).

13. Dio Chrys. Or. 1.52-58. Vgl. Moles (1990) 318-319.

14. Fitzgerald/White (1983) 141 Anm.22. Siehe außerdem Sil. Pun. 15.32,33 und 15.108.

15. Alexis frg. 140 PCG.

16. Whitmarsh (2001) 122.

17. Humble/Sidwell (2006) 219.

18. Humble/Sidwell (2006) 222.

19. Vgl. auch die Bevorzugung des „weiblichen“ Rhetoriklehrers gegenüber dem „männlichen“ in Lukian. 41.9 und 11. Siehe Cribiore (2007) 72.

20. Kuntz (1993-94), Davies (2003), Davies (2004).

21. Über den Zusammenhang bereits in Hom. Il. 24.28-29 siehe Davies (1981).

22. Buffière (1973) 330-331.

23. Apul. Met. 10.29-33.

24. Finkerpearl (1991) 222 und Zimmermann-de Graaf (1993) 151-152.

25. Apul. Met. 10.33 .

26. Kossatz-Deissmann (1994) 186.

27. Zuerst in Kratinos' Dionysalexandros (PCG IV frg. 39-51), dann Eur. Troad. 971-974, Isocr. Helena 41, Apollod. Ep. 3.2, Hygin. 92, Lukian. 35.11-13, Apul. Met. 30-32.

28. Ich danke Alexander Riddiford (Oxford) dafür, mir diesen Hinweis gegeben und mir seine englische Übersetzung des Originaltextes zur Verfügung gestellt zu haben.

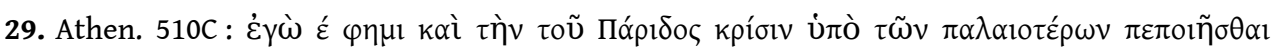

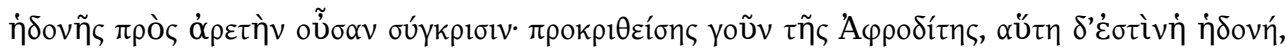

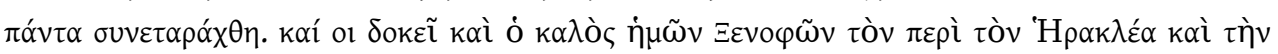

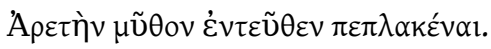

30. Vgl. Elsner (2007) 1.

31. Kossatz-Deissmann (1994) 186.

32. Lukian. 35.11, Apul. Met. 10.30-31, Anth. Graec. 9.633, 619, 637 ; 16.165, 169, 170, 178, 181, 182. Zur bildenden Kunst siehe Kossatz-Deissmann (1994) 176-177.

33. Rufin. Ep. 11 und 12 Page.

34. Hedreen (2001) 186 und 209-211.

35. Stinton (1965) 15.

36. Cratin. frg. 39-51 PCG, Eur. Troad. 971-974, Isocr. Helena 41, Apollod. Ep. 3.2, Hygin. 92, Lukian. 35.11-13, Apul. Met. 30-32.

37. Stafford (2005) 76.

\section{RÉSUMÉS}

In some versions of the story of Heracles at the crossroads, his decision is based exclusively on the visual aspects of the two options, incorporated in two female figures (most notably in Dio Chrysostom's first Kingship Oration). The women do not explain in speeches what they represent; it is only by their appearance that their moral significance is highlighted. This concentration on the visual aspects of the choice reflects the influence of the story of the judgement of Paris. Both myths have in common that the choice described does not reveal new 
insights into the different options on offer, but rather into the moral qualities of the one who chooses.

INDEX

Mots-clés : Dio Chrysostomos, Lebenswahl, Herakles am Scheideweg, Parisurteil, Visualität

\section{AUTEUR}

ANDREA HARBACH

Université de Genève

andrea.harbach@unige.ch 